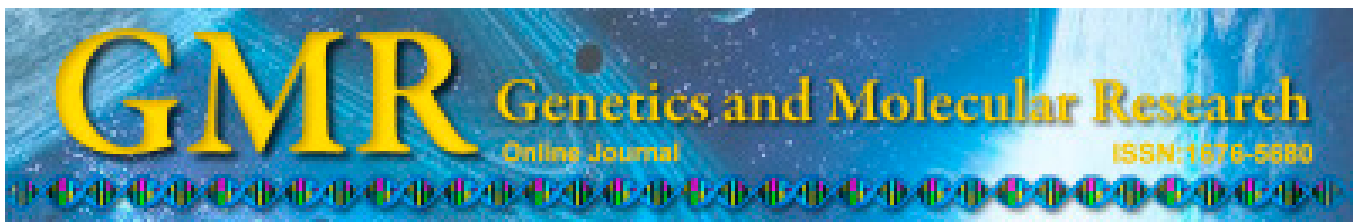

\title{
Expression and role of cyclophilin B in stomach cancer
}

\author{
D.Q. Meng', P.L. $\mathrm{Li}^{2}$ and M. Xie \\ ${ }^{1}$ Department of Gastroenterology, Jinan Sixth Hospital, Zhangqiu, China \\ ${ }^{2}$ Central South University, Changsha, China \\ ${ }^{3}$ Zunyi Medical College, Zunyi, China \\ Corresponding author: D.Q. Meng \\ E-mail: mengdeqindg@163.com
}

Genet. Mol. Res. 14 (2): 5346-5354 (2015)

Received May 12, 2014

Accepted November 4, 2014

Published May 22, 2015

DOI http://dx.doi.org/10.4238/2015.May.22.5

ABSTRACT. We elucidated the expression of ciclosporin protein B (cyclophilin B) in stomach cancer tissue and the correlation between cyclophilin B and clinicopathological parameters, and determined the effect of cyclophilin B on growth and proliferation of stomach cancer cells. Pathological sections of stomach cancer and paracancerous tissue were collected for detecting the expression and distribution of cyclophilin B, using immunohistochemistry, and for analyzing the relationship between the expression levels of cyclophilin B in stomach cancer and the clinical pathological parameters of the patients. A cyclophilin BsiRNA lentiviral (LV-cyclophilin B-siRNA) and corresponding control vector (LV-siRNA-con) were constructed. MTT and cell cycle assays were used to detect the effect of downregulation of cyclophilin B expression on in vitro growth and proliferation and clone formation capacity of BGC823 and SGC7901 cells. The cyclophilin B-positive rate of stomach cancer tissue was 84.29\% (59/70) and that of paracancerous tissue was 56.00\% (28/50). The expression of cyclophilin B in stomach cancer tissue was significantly higher than that in paracancerous tissue $(\mathrm{P}<0.05)$. Staining for cyclophilin B was primarily present in the cytoplasm and was seldom present in the cell 
nuclei. Downregulation of cyclophilin B significantly inhibited growth and proliferation of stomach cancer cells, cell cycle progression, and in vivo tumorigenicity capacity. Cyclophilin $\mathrm{B}$ has a high diagnostic value for stomach cancer and its downregulation can effectively inhibit the growth of stomach cancer cells. Thus, cyclophilin B may be a potential therapeutic target for stomach cancer treatment.

Key words: Cell proliferation; Ciclosporin protein B (cyclophilin B); Stomach cancer

\section{INTRODUCTION}

Stomach cancer impacts human health severely, with more than 100 cases of stomach cancer newly diagnosed globally every year. Stomach cancer ranks fourth in morbidity and third in mortality among tumors (Liu et al., 2013). The effect of therapy in stomach cancer is closely correlated with the stage of the disease, the therapeutic method used, and the administration of therapy. More than $90 \%$ of patients diagnosed with early gastric cancer can survive for more than 5 years or undergo cure, whereas the survival rate of patients with late stomach cancer is less than 5\% over 5 years (Ferronha et al., 2012). Thus, early diagnosis is crucial for improving the curative effect of therapy in stomach cancer and for elevating the survival rate. The majority of stomach cancer patients report to a doctor when they feel physically uncomfortable. Stomachoscopy is not generally used, but most stomach cancer patients only show symptoms in the middle and advanced stages. Currently, the tumor markers that are clinically used for stomach cancer screening primarily include CEA, CA19-9, CA72-4, and ELCOX; however, these markers have certain limitations (Gan et al., 2013). Therefore, it is important to develop new serological tumor markers for stomach cancer.

Ciclosporin protein B (cyclophilin B) was so named because it binds to cyclosporin A (CsA), which facilitates immunosuppression (Väkevä et al., 2008). Cyclophilin B is a secreted protein and can be detected in vivo in prokaryotic and eukaryotic organisms (Li et al., 2013). In recent years, this protein has been shown to be highly expressed in breast, liver, colon, and pancreatic cancer and to play an important role in the malignant progression of tumors (Kim et al., 2012). To date, there have been no reports on cyclophilin B in stomach; hence, we here aimed to investigate its expression and role in stomach cancer and to explore its potential value in diagnosing or treating stomach cancer.

\section{MATERIAL AND METHODS}

\section{Reagent and instruments}

The anti-cyclophilin B antibody (Fusheng Biotech, Shanghai, China); the Real Envision Detection kit (Boyan, Shanghai, China); DMEM and RPMI 1640 (Ruixiang, Beijing, China); fetal bovine serum (Biowish, Hangzhou, China); carbon dioxide incubator (SouthNorth Instrument \& Equipment, Zhengzhou, China); ELISA monitor (PeproTech,USA); flow cytometer (Hach,USA); the optical microscope (Olympus, Japan). 


\section{Tissue specimens}

Paraffin sections (70, 50, and 30 samples of stomach cancer tissue, paracancerous tissue, and normal gastric tissue) were obtained from the Pathology Department of Jinan Sixth Hospital, from January 2013 to December 2013. Of the 70 stomach cancer patients, 43 were men and 27 were women.

\section{Immunohistochemical staining}

The tissue sections were dewaxed, hydrated, washed with phosphate-buffered saline (PBS) for $5 \mathrm{~min}$, thrice, incubated for $30 \mathrm{~min}$ in $80 \%$ methanol containing $3 \% \mathrm{H}_{2} \mathrm{O}_{2}$, in the dark. Sections were then blocked in $2 \%$ normal goat serum at $37^{\circ} \mathrm{C}$ for $1 \mathrm{~h}$, and then overnight at $4^{\circ} \mathrm{C}$ after drop-wise addition of anti-cyclophilin B polyclonal antibody (diluted 1:250). Subsequently, sections were incubated for $30 \mathrm{~min}$ at room temperature with Solution A (secondary antibody) of the Real Envision Detection System. To determine the degree of staining, $50-100 \mu \mathrm{L}$ of freshly prepared DAB solution was added to the sections drop-wise, after which the sections were washed for approximately $15 \mathrm{~min}$ under tap water to terminate staining. Sections were then restained with hematoxylin, washed for approximately 15 min under tap water, dehydrated with a graded alcohol series, clarified with dimethylbenzene solution, sealed, and then examined under the microscope.

\section{Immunohistochemical staining evaluation and statistical analysis}

The immunohistochemical results of the tissue sections were evaluated by two pathologists blinded to the clinical pathological data. Under the microscope, 5200 fields of view were taken randomly. The percentage of positive staining was obtained by calculating the ratio between the positive tumor cells and all tumor cells throughout the whole section. Each specimen was scored as Grade $0-4$, with 0 indicating negative; 1 indicating positive staining from 1 to $25 \%$; 2 indicating positive staining from 26 to $50 \%$; 3 indicating positive staining from 51 to $75 \%$; and 4 indicating positive staining from 76 to $100 \%$. The staining intensity was quantified as 1 (negative), 2 (weakly positive), 3 (moderately positive), and 4 (strongly positive). The staining for each section was evaluated on the basis of the sum of the two parameters given above and 0 was evaluated as negative (-), 1-2 was evaluated as weakly positive ( \pm ), 3-5 as positive $(+)$, and 6-8 as strongly positive $(++)$. The positivity rate referred to the ratio of the sum of the weakly positive, positive, and strongly positive cases to the total number of cases (Li et al., 2014).

\section{Construction of cyclophilin BsiRNA lentiviral vector}

The cyclophilin BsiRNA primer sequence was 5'-AGACAGGTGGAGAGTTCACCA-3' and the vector was GV115. A single-stranded DNA oligonucleotide containing an interference sequence was first synthesized chemically. Then, a double-stranded oligonucleotide was generated after annealing and pairing. This was cloned into the digested RNAi lentiviral vector via the $A g e \mathrm{I}$ and $E c o$ RI sites. The ligation products were transferred to appropriately prepared bacterial competent cells; positive clones were identified by PCR and sequenced. Construction of lentiviral vectors was carried out as previously described (McIntosh et al., 2012). 


\section{Establishing a stable cell line with a low expression level of cyclophilin B}

Cells $\left(3 \times 10^{3}\right)$ were seeded on a 96-well microplate. The stock solution of virus, at a titer of $10^{8}$, was diluted $10 \mathrm{X}$ and $100 \mathrm{X}$. Of these three virus solutions, $10 \mu \mathrm{L}$ was added to 3 individual wells, representing MOIs of 100, 10, and 1, respectively. Fluorescence intensity was observed after $72 \mathrm{~h}$ to confirm that the MOI used in the experiment was 10 .

SGC7901 and BGC823 cells were inoculated on a 24-well microplate, at 4 x 10 $10^{4}$ cells/ well. The virus was centrifuged and diluted 10X. Of this diluted virus solution, $10 \mu \mathrm{L}$ was added $(\mathrm{MOI}=10)$; as a control, virus from the culture solution was used. The culture solution was replaced after the cells were incubated overnight. After reaching confluence, the cells were passaged to a 6-well microplate, transferred to a $25-\mathrm{cm}$ culture flask, and eventually cryopreserved for later use. The stable cell lines obtained in this way are termed SGC7901siRNA, SGC7901-con, BGC823-siRNA, and BGC823-con.

\section{MTT experiment}

Single-cell suspensions of BGC823-si, BGC823-con, BGC823, SGC7901-si, SGC7901-con, and SGC7901 cells were prepared in RPMI 1640 medium containing 10\% fetal bovine serum. These cell solutions were placed in the wells of a 96-well microplate, with 1000 cells/well, in a volume of $200 \mu \mathrm{L}$. MTT solution $(20 \mu \mathrm{L}$ of $5 \mathrm{mg} / \mathrm{mL}$ solution) was added to each well after the cells had been cultured for 3 days. The cultures were then incubated at $37^{\circ} \mathrm{C}$ for $4 \mathrm{~h}$, after which the supernatants were carefully removed. Dimethyl sulfoxide solution $(150 \mu \mathrm{L})$ was added to each well and the plates agitated for $10 \mathrm{~min}$ to ensure that the formazan crystals dissolved completely. The light absorption value of each well was determined using an ELISA reader at a wavelength of $490 \mathrm{~nm}$. The growth curves of various groups of cells were plotted, with time as the abscissa and the average absorption value of 3 triplicate wells as the ordinate (Yang et al., 2013a).

\section{Analysis of cell cycle}

Experimental cells (transfection group, control group, and blank group; 1 x $10^{6}$ cells) were incubated on a $60-\mathrm{mm}$ microplate, digested with pancreatic enzyme, and the products collected after $48 \mathrm{~h}$. Samples were washed twice with cold PBS and mixed by pipetting, after which the supernatant was discarded and $1 \mathrm{~mL}$ of $70 \%$ pre-cooled alcohol was added. Samples were then refrigerated at $4^{\circ} \mathrm{C}$ for more than $12 \mathrm{~h}$. The cells were washed with PBS to remove alcohol, centrifuged at $1000 \mathrm{rpm}$ for $5 \mathrm{~min}$, and then again washed twice. The cells were subsequently re-suspended in $0.5 \mathrm{~mL}$ PBS. Propidium iodide and RNaseA were added to a final concentration of $50 \mathrm{~g} / \mathrm{mL}$. The mixture was incubated in a warm bath at $37^{\circ} \mathrm{C}$ for $30 \mathrm{~min}$. Cell cycle was then detected using a flow cytometer (Martin et al., 2007).

\section{Statistical analysis}

The SPSS 16.0 software was used for statistical analysis. The $\chi^{2}$ test was used to analyze the relationship between immunohistochemical staining and clinical pathological factors. $\mathrm{P}<0.05$ indicated that the difference was significant statistically. 


\section{RESULTS}

\section{Immunohistochemical staining results}

Immunohistochemical staining indicated that cyclophilin B was primarily present in the cytoplasm and was seldom present in the cell nuclei. In normal liver tissue, cyclophilin B stained weakly positively or negatively in the cytoplasm and karyon. However, there was partial positive staining for cyclophilin B in inflammatory cells in the interstitial tissue (Figure 1).

A

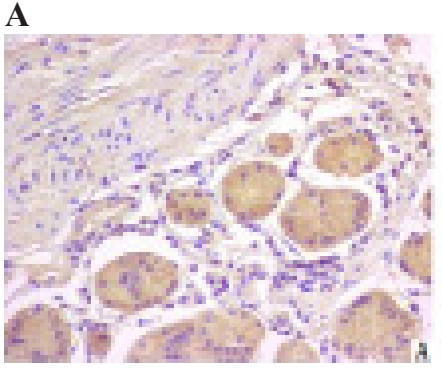

B

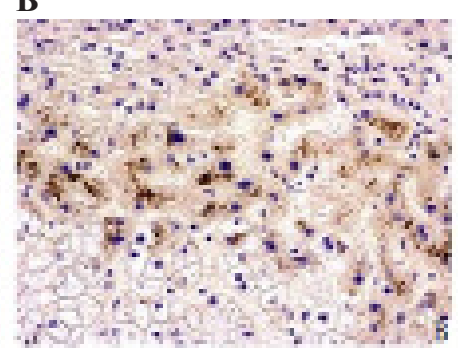

C

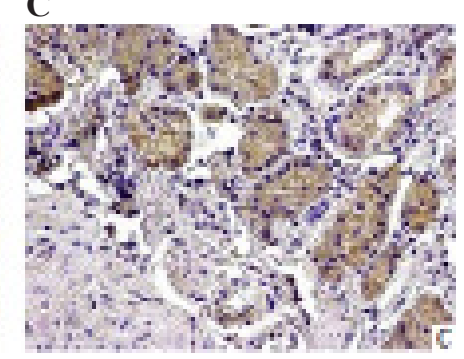

Figure 1. Cyclophilin B staining in stomach cancer and paracancerous tissues. A. Normal tissue; B. paracancerous tissue; C. stomach cancer tissue.

\section{Expression of cyclophilin B in stomach cancer, paracancerous tissues, and normal gastric tissue}

Immunohistochemical staining indicated that the level of cyclophilin B varied in stomach cancer and paracancerous tissues. Of the 70 stomach cancer tissue specimens, 11 were negative for cyclophilin B expression, 18 were weakly positive, 26 were positive, and 15 were strongly positive. The positivity rate for cyclophilin B in stomach cancer tissue was $84.29 \%(59 / 70)$. Of the 50 paracancerous tissue specimens, 22 were negative, 9 were weakly positive, 13 were moderately positive, and 6 were strongly positive for cyclophilin B expression. The positivity rate for cyclophilin B in paracancerous tissue was $56.00 \%(28 / 50)$. The expression of cyclophilin B in stomach cancer tissue was statistically significantly higher than that in paracancerous tissue $(\mathrm{P}<0.05$; Table 1$)$.

Table 1. Expression of cyclophilin B in stomach cancer and paracancerous tissues.

\begin{tabular}{lcccccc}
\hline & No. of cases & \multicolumn{4}{c}{ Expression of cyclophilin B } & P value \\
\cline { 3 - 6 } & & - & \pm & + & ++ & 0.017 \\
\hline Stomach cancer tissue & 70 & 11 & 18 & 26 & 15 & \\
Paracancerous tissue & 50 & 22 & 9 & 13 & 6 & \\
\hline
\end{tabular}

\section{Clinicopathological parameters of patients}

Expression of cyclophilin B and the clinicopathological data of 70 patients with stomach cancer were analyzed for correlation. The expression levels of cyclophilin B in stomach cancer tissue was associated with $\mathrm{T}$ staging and lymphatic metastasis of stomach cancer, but 
not age, gender, and degree of differentiation. Indeed, the positive staining rate of cyclophilin B differed little in stomach cancer tissues with different degrees of differentiation. However, the positivity rate at Stage T3 was significantly higher than that at Stages T1 or T2 $(\mathrm{P}<0.01)$. The positivity rate for cyclophilin B staining in the stomach cancer tissue with lymphatic node metastasis was also significantly higher than for that without lymphatic node metastasis. These correlations were statistically significant $(\mathrm{P}<0.05$; Table 2$)$.

Table 2. Correlation between the expression levels of cyclophilin B and clinicopathological parameters of
patients with stomach cancer.

\section{Cell growth and proliferation as determined by MTT assay}

After infection with the cyclophilin B-siRNA lentiviral vector, BGC823 and SGC7901 cells grew significantly slower from day 4 and day 5 , respectively, as compared with the blank control group and the random sequence group (all $\mathrm{P}<0.05$ ). There was no statistically significant difference between the blank control group and the interference sequence group (Figure 2).

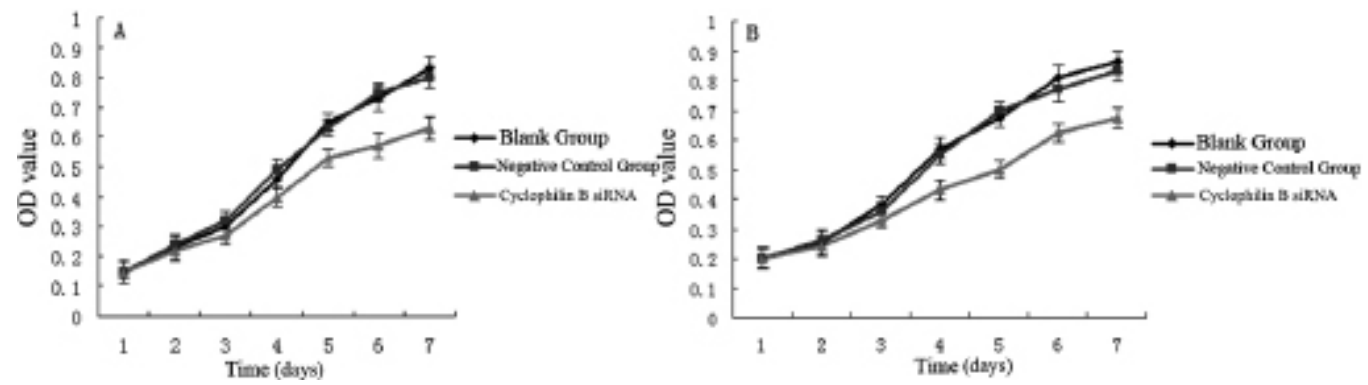

Figure 2 Cell growth and proliferation as determined by MTT assay. A. Cell growth after BGC823 cells were infected with cyclophilin B siRNA; B. cell growth after SGC7901 cells were infected with cyclophilin B siRNA. The cell growth rate slowed from days 4 and 5 for A and B, respectively, compared with the blank group and the negative control group $(\mathrm{P}<0.05)$. 


\section{Cell cycle}

After BGC823 and SGC7901 cells had been transfected with cyclophilin BsiRNA, the proportion of cells at the G1/S stage decreased significantly, as compared with those transfected with cyclophilin B-con and the blank control group that had not been transfected with any vector $(\mathrm{P}<0.05)$. Cyclophilin BsiRNA inhibited the progression of the cell cycle and caused arrest of stomach cancer cells at the G1/S stage, as shown in Figure 3.
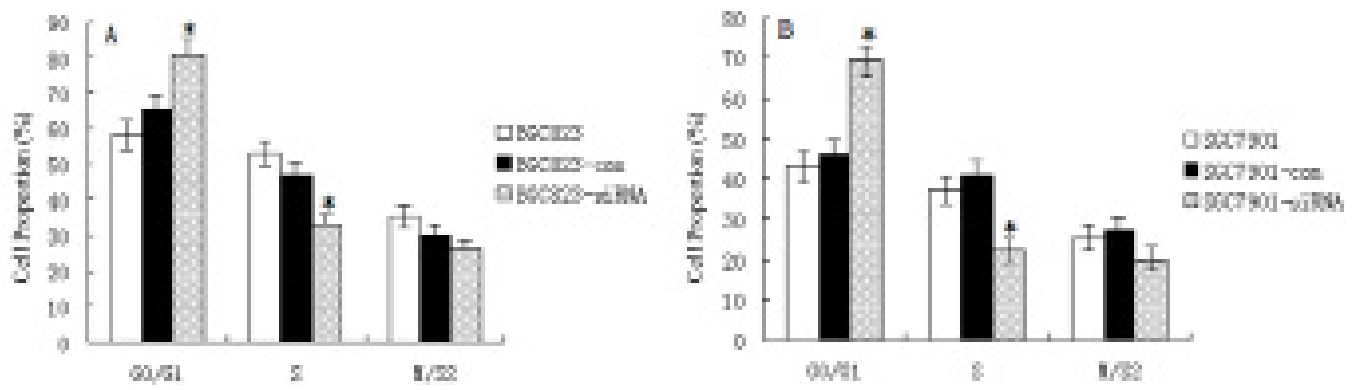

Figure 3 Effect of cyclophilin B on the cell cycle. A. Effect on the BGC823 cell cycle after downregulation of cyclophilin B; B. effect on the SGC7901 cell cycle after downregulation of cyclophilin B. The proportion of the cells at the G1 stage was significantly higher than that in the blank group and the negative control group, while the cells at the $\mathrm{S}$ stage decreased significantly $(\mathrm{P}<0.05)$ after downregulation of cyclophilin $\mathrm{B}$.

\section{DISCUSSION}

Stomach cancer is one of the most common malignant tumors. Based on statistics from the World Health Organization, $70 \%$ of newly diagnosed stomach cancer cases and deaths resulting from stomach cancer occur in developing countries. The morbidity in male patients is twice that in female patients; and the death of $40 \%$ patients with stomach cancer is preventable (Mima et al., 2013). The regions with the highest morbidity of stomach cancer are East Asia, Eastern Europe, and South America, whereas it is very low in most areas in North America and Africa (Ji and Jiang, 2013). The regional differences in morbidity may be associated with dietary habits and Helicobacter pylori infection (Alfizah et al., 2013). Smoking, a high-salt diet, and smoked food are common life style and environmental factors that give rise to stomach cancer; overweight and obesity are also risk factors for stomach cancer (Navarro Silvera et al., 2014). Moreover, in recent studies, it has been reported that binge drinking is significantly associated with stomach cancer. In contrast, fresh vegetables and fruits have antistomach cancer effects (Lee and Derakhshan, 2013; Zhang et al., 2013). A number of cancer prevention guides, changes in life style, and avoidance of the risk factors mentioned above seem to be the most effective and simplest methods for reducing the morbidity and mortality of stomach cancer (Ni et al., 2010).

In addition to these external factors, research has shown that multiple genes (activation of cancer-promoting genes, reduction of the expression of or loss of function of cancersuppressor genes) and abnormalities of various signal transduction pathways play an important role in the occurrence, progression, invasion, and metastasis of stomach cancer (Akhtar et al., 2013; Wang et al., 2013). The vascular endothelial growth factor receptor (VEGFR) is closely associated with such biological behaviors, such as the occurrence, infiltration, endo- 
thelial cell proliferation, metastasis, etc., of tumor vessels (Hwang et al., 2013). In stomach cancer, the expression of VEGFR is also closely associated with lymph node metastasis. High levels of expression of this receptor in stomach cancer cells may predict a poor prognosis for the patient, while inhibition of its activity may serve as an important treatment approach for stomach cancer (Ozmen et al., 2011). Proteins related to regulation of the cell cycle also participate in such processes as proliferation, differentiation, etc., of stomach cancer cells (Yang et al., 2013b). Cell cycle-dependent protein kinases (CDKs) include CDK1, CDK4, cyclinD1, cycline, etc.; these proteins regulate the cell cycle and may promote cell proliferation by regulating shortening of the cell cycle (Zheng et al., 2013).

Cyclophilin was first identified as a protein binding to the immunosuppressive drug, CsA. The proteins belonging to this family contain two structural domains: one is the cyclophilin structural domain, which exists widely in the various family members, while the other, variable, structural domain exerts effects on subcellular positioning and division of roles between family members (Ishikawa and Bachinger, 2013). The Cyp family is highly conserved throughout evolution, which indicates that it plays an important role and is a housekeeping gene. Human cyclophilin includes 7 types: CypA, cyclophilin B, CypC, CypD, CypE, Cyp40, and CypNKCyp; these proteins are subcellularly widely distributed, including the cytoplasm, endoplasmic reticulum, mitochondria, and cell nucleus (Torresan et al., 2008). cyclophilin B is closely associated with the occurrence and progression of tumors. Research has demonstrated that cyclophilin B has a higher expression level in patients with colon cancer than in healthy people and it plays an important role in the early diagnosis of pancreatic cancer (Lim et al., 2013). Both stomach cancer and pancreatic cancer are intestinal tumors, and may therefore share tumor markers. Our study also demonstrated that the expression level of cyclophilin B in stomach cancer is significantly higher than that in paracancerous tissue, indicating that it may be closely associated with the occurrence and progression of stomach cancer. In recent years, it has been found that cyclophilin B is overexpressed in liver, colon, pancreatic, and breast cancer (Chen and Wang, 2013; Wen et al., 2013).

The lentiviral vector allows highly efficient infection of cells, irrespective of whether they are at the division stage or not, and allows facile establishment of a stable transfected cell line (Qin et al., 2011). It has been reported that downregulation of cyclophilin B expression can inhibit the growth, proliferation, and metastasis of cells (Ada et al., 2010). In our study, BGG823 and SGC7901 were infected by constructing a lentivirus siRNA vector. MTT and cell cycle assays were conducted to evaluate the effect of downregulation of cyclophilin B expression on the growth and proliferation of stomach cancer cells. Our results showed that downregulation of cyclophilin B in this way slowed the growth and proliferation of stomach cancer cells, arresting the cell cycle; this would lead to a significant decrease in the in vivo tumorigenicity capacity of these cells.

\section{REFERENCES}

Ada AO, C Kunak S, Hancer F, Bilgen S, et al. (2010). CYP and GST polymorphisms and survival in advanced non-small cell lung cancer patients. Neoplasma 57: 512-521.

Akhtar J, Wang Z, Zhang ZP and Bi MM (2013). Lentiviral-mediated RNA interference targeting stathmin1 gene in human gastric cancer cells inhibits proliferation in vitro and tumor growth in vivo. J. Transl. Med. 11: 212-215.

Alfizah H, Noraziah MZ, Chao MY, Rahman MM, et al. (2013). Helicobacter pylori: molecular detection of vacA gene and vacuolating activity in human gastric adenocarcinoma cells. Clin. Ter. 164: 301-305.

Chen C and Wang DW (2013). CYP epoxygenase derived EETs: from cardiovascular protection to human cancer therapy. 
Curr. Top. Med. Chem. 13: 1454-1469.

Ferronha I, Bastos A and Lunet N (2012). Prediagnosis lifestyle exposures and survival of patients with gastric cancer: systematic review and meta-analysis. Eur. J. Cancer Prev. 21: 449-452.

Gan R, Liu X, Zhou Y, Tan Y, et al. (2013). Cloning and expression analysis of the gastric carcinoma-related gene, ELCOX3. Oncol. Lett. 6: 1744-1748.

Hwang JE, Lee JH, Park MR, Kim DE, et al. (2013). Blockade of VEGFR-1 and VEGFR-2 enhances paclitaxel sensitivity in gastric cancer cells. Yonsei Med. J. 54: 374-380.

Ishikawa Y and Bachinger HP (2013). An additional function of the rough endoplasmic reticulum protein complex prolyl 3-hydroxylase 1 cartilage-associated protein. Cyclophilin B: the CXXXC motif reveals disulfide isomerase activity in vitro. J. Biol. Chem. 288: 31437-31446.

Ji W and Jiang Z (2013). Effect of shRNA-mediated inhibition of Nanog gene expression on the behavior of human gastric cancer cells. Oncol. Lett. 6: 367-374.

Kim K, Kim H, Jeong K, Jung MH, et al. (2012). Release of overexpressed CypB activates ERK signaling through CD147 binding for hepatoma cell resistance to oxidative stress. Apoptosis 17: 784-796.

Lee YY and Derakhshan MH (2013). Environmental and lifestyle risk factors of gastric cancer. Arch. Iran. Med. 16: 358-365.

Li L, Yang F, Wang X, Hu J, et al. (2014). Effect of 15-hydroxyprostaglandin dehydrogenase gene on the proliferation of gastric cancer cell murine forestomach carcinoma. Exp. Ther. Med. 7: 290-294.

Li Z, Zhang D, Gao J, Li WX, et al. (2013). Cyclosporine combined with nonlytic interleukin 2/Fc fusion protein improves immune response to hepatitis B vaccination in a mouse skin transplantation model. Transplant. Proc. 45: 2559-2564.

Lim SM, Hwang JW, Ahn JB, Bae SK, et al. (2013). Combination of CYP inhibitor with MEK/ERK inhibitor enhances the inhibitory effect on ERK in BRAF mutant colon cancer cells. Anticancer Res. 33: 2499-2508.

Liu JW, He CY, Sun LP, Xu Q, et al. (2013). The DNA Repair Gene ERCC6 rs1917799 Polymorphism is Associated with Gastric Cancer Risk in Chinese. Asian Pac. J. Cancer Prev. 14: 6103-6108.

Martin BT, Kleiber K, Wixler V, Raab M, et al. (2007). FHL2 regulates cell cycle-dependent and doxorubicin-induced p21Cip1/Waf1 expression in breast cancer cells. Cell Cycle 6: 1779-1788.

McIntosh JH, Cochrane M, Cobbold S, Waldmann H, et al. (2012). Successful attenuation of humoral immunity to viral capsid and transgenic protein following AAV-mediated gene transfer with a non-depleting CD4 antibody and cyclosporine. Gene Ther. 19: 78-85.

Mima K, Fukagawa T, Kurashige J, Takano Y, et al. (2013). Gene expression of bone morphogenic protein 8B in the primary site, peripheral blood and bone marrow of patients with gastric cancer. Oncol. Lett. 6: 387-392.

Navarro Silvera SA, Mayne ST, Gammon MD, Vaughan TL, et al. (2014). Diet and lifestyle factors and risk of subtypes of esophageal and gastric cancers: classification tree analysis. Ann. Epidemiol. 24: 50-57.

Ni XF, Wu CP and Jiang JT (2010). Serum VEGFR-3 and survival of advanced gastric cancer patients treated with FOLFOX. World J. Gastroenterol. 16: 2163-2169.

Ozmen F, Ozmen MM, Ozdemir E, Moran M, et al. (2011). Relationship between LYVE-1, VEGFR-3 and CD44 gene expressions and lymphatic metastasis in gastric cancer. World J. Gastroenterol. 17: 3220-3228.

Qin XJ, Dai DJ, Gao ZG, Huan JL, et al. (2011). Effect of lentivirus-mediated shRNA targeting VEGFR-3 on proliferation, apoptosis and invasion of gastric cancer cells. Int. J. Mol. Med. 28: 761-768.

Torresan C, Oliveira MM, Torrezan GT, de Oliveira SF, et al. (2008). Genetic polymorphisms in oestrogen metabolic pathway and breast cancer: a positive association with combined CYP/GST genotypes. Clin. Exp. Med. 8: 65-71.

Väkevä L, Reitamo S, Pukkala E, Sarna S, et al. (2008). Long-term follow-up of cancer risk in patients treated with shortterm cyclosporine. Acta Derm. Venereol. 88: 117-120.

Wang MY, Zhu ML, He J, Shi TY, et al. (2013). Potentially functional polymorphisms in the CASP7 gene contribute to gastric adenocarcinoma susceptibility in an eastern Chinese population. PLoS One 8: 4041-4045.

Wen H, Feng CC, Fang ZJ, Xia GW, et al. (2013). Study on bladder cancer susceptibility and genetic polymorphisms of XPC, XPG, and CYP in smokers and non-smokers. Actas Urol. Esp. 37: 259-265.

Yang Q, Gao J, Xu L, Zeng Z, et al. (2013a). Promoter hypermethylation of BCL6B gene is a potential plasma DNA biomarker for gastric cancer. Biomarkers 18: 721-725.

Yang XB, Zhao JJ, Huang CY, Wang QJ, et al. (2013b). Decreased expression of the FOXO3a gene is associated with poor prognosis in primary gastric adenocarcinoma patients. PLoS One 8: 158-162.

Zhang J, Zhan Z, Wu J, Zhang C, et al. (2013). Association among polymorphisms in EGFR gene exons, lifestyle and risk of gastric cancer with gender differences in Chinese Han subjects. PLoS One 8: 254-258.

Zheng Q, Zhao LY, Kong Y, Nan KJ, et al. (2013). CDK-associated Cullin 1 can promote cell proliferation and inhibit cisplatin-induced apoptosis in the AGS gastric cancer cell line. World J. Surg. Oncol. 11: 263-268. 